\title{
Conformation and Assembly of Polypeptide Scaffolds in Templating the Synthesis of Silica: An Example of a Polylysine Macromolecular "Switch"
}

Siddharth V. Patwardhan, $*^{\dagger \S \rrbracket}$ Ronak Maheshwari, ${ }^{\S}$ Niloy Mukherjee, ${ }^{\dagger}$ Kristi L. Kiick* ${ }^{\S}$ and Stephen J. Clarson* $*^{\dagger}$

${ }^{\dagger}$ Department of Chemical and Material Engineering, University of Cincinnati, Cincinnati, OH 452210012, USA. ${ }^{\S}$ Department of Materials Science and Engineering, University of Delaware, Newark, DE 19716, USA. " Division of Chemistry, School of Biomedical and Natural Sciences, Nottingham Trent University, Nottingham, NG11 8NS, UK.

*Siddharth.Patwardhan@ntu.ac.uk, Kiick@udel.edu, Stephen.Clarson@uc.edu

Figure S1 PLL-mediated silica formation under static conditions using TMOS. (a) SEM of spherical silica particles synthesised, insert shows the EDS spectrum (Bar $=500 \mathrm{~nm}$ ). (b) TEM of spherical silica particles synthesised, the top left insert shows the SAED pattern (Bar $=500 \mathrm{~nm}$ ). (c) AFM of spherical silica particles synthesised and line scans (image size $=5 \mu \mathrm{m} \times 5 \mu \mathrm{m}$ ).
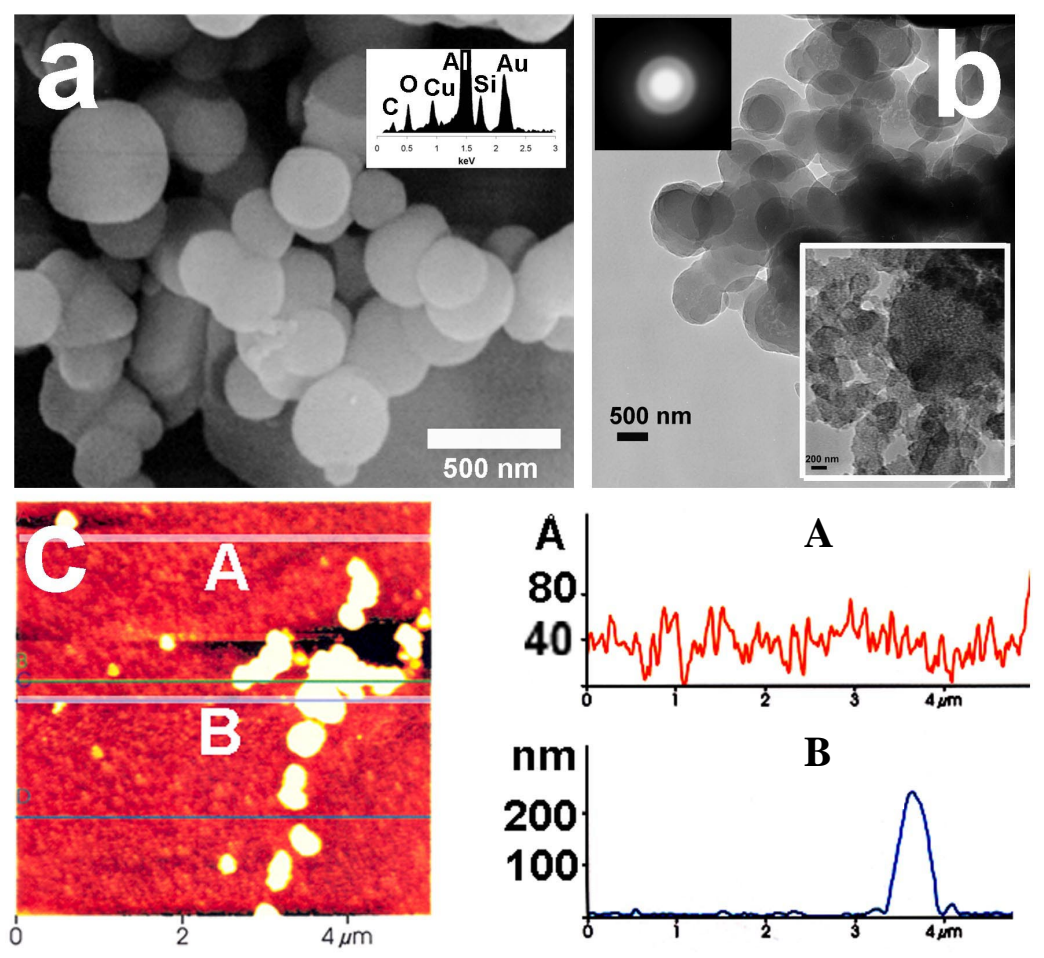
Figure S2 Thermogravimetric analysis of PLL-silica hybrid sample.

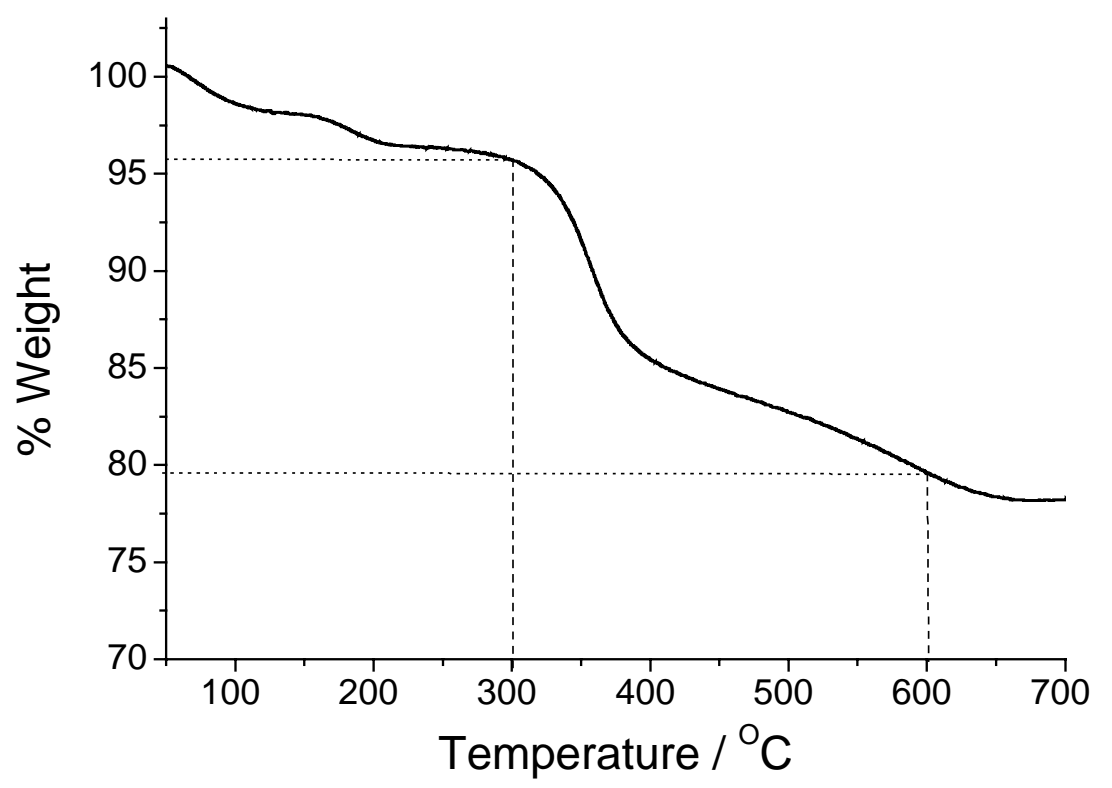

Figure S3 Deconvoluted silica peaks in the IR spectra of silica samples before and after calcination. LO $=$ longitudinal optical and $\mathrm{TO}=$ transverse optical.

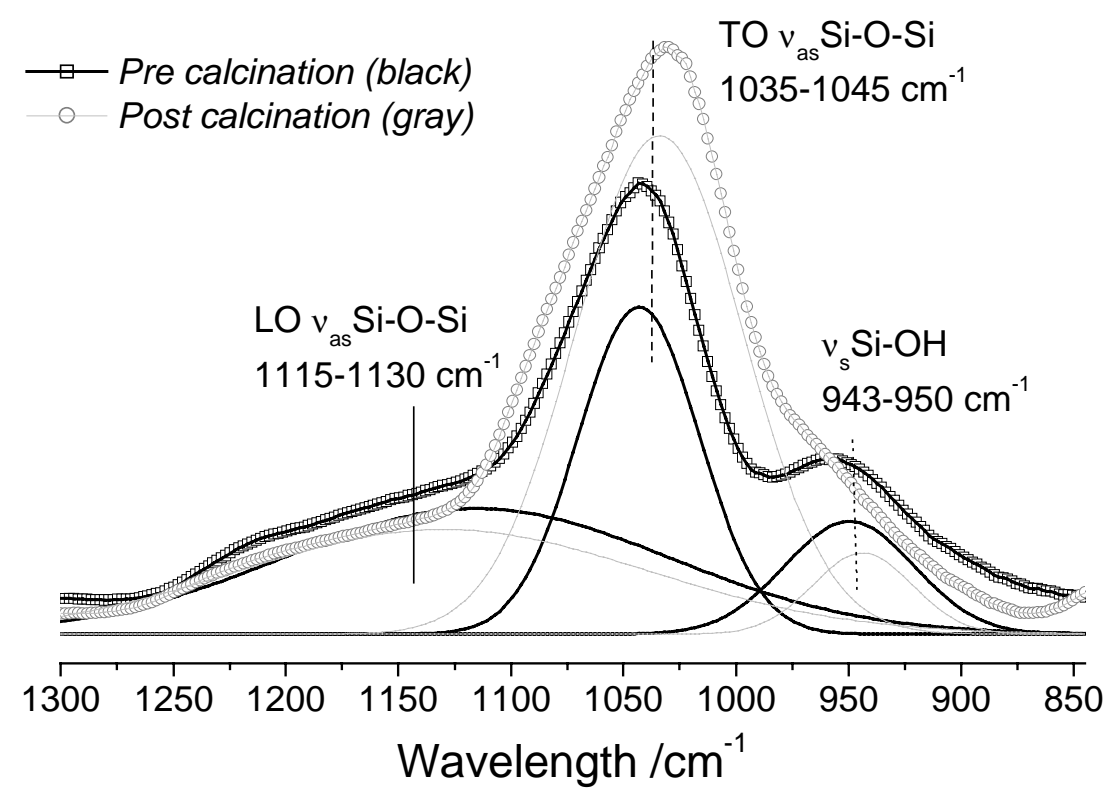

